\title{
The interaction of hCG, hydroxysteroids and interstitial fluid on rat Leydig cell steroidogenesis in vitro
}

\author{
G. P. Risbridger, G. Jenkin and D. M. de Kretser \\ Departments of Anatomy and Physiology, Monash University, Clayton, Victoria 3168, Australia
}

\begin{abstract}
Summary. Rat testicular interstitial fluid and hydroxycholesterol both stimulated testosterone production by isolated Leydig cells in vitro in a dose-dependent manner, but the dose-response lines were not parallel. The addition of cycloheximide blocked the stimulation by interstitial fluid but not that of hydroxycholesterol. Use of the com-

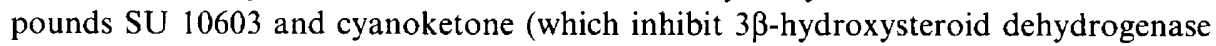
and $17 \alpha$-hydroxylase respectively) or aminoglutethimide (which acts on the cholesterol side-chain cleavage enzyme) showed that the stimulatory factor(s) in interstitial fluid stimulated steroidogenesis at the cholesterol side-chain cleavage enzyme, before the conversion of pregnenolone. This enzyme is rate-limiting in the synthesis of testosterone by Leydig cells and a site of action of $\mathrm{LH}$; therefore, these results support the view that an interstitial fluid factor may be involved in the paracrine regulation of testicular steroidogenesis.
\end{abstract}

\section{Introduction}

The production of testosterone by Leydig cells is stimulated by pituitary LH. However, there is increasing evidence to suggest that intratesticular concentrations of testosterone are regulated by local factors which may emanate from the seminiferous tubules and be transferred across the basement membrane into interstitial fluid to affect the Leydig cells (see reviews by de Kretser, 1982; Parvinen, 1982; Sharpe, 1983). In accordance with this proposal it has been demonstrated that rat testicular interstitial fluid (which bathes the Leydig cells in vivo) contains a proteinaceous factor(s), which is not $\mathrm{LH}$, and can stimulate testosterone production in vitro by isolated Leydig cells from adult male rats (Sharpe \& Cooper, 1984; Risbridger et al., 1986).

Interstitial fluid may contain a substrate (such as cholesterol) not present in incubation media which could be utilized by the Leydig cells for testosterone production, or the factor(s) in interstitial fluid may stimulate a specific step in steroidogenesis to control the testosterone concentrations within the testis.

The aim of the present study was to investigate the mechanism of action of interstitial fluid on isolated Leydig cells in vitro by using hydroxycholesterol and inhibitors of some of the enzymes involved in the conversion of cholesterol to testosterone.

\section{Materials and Methods}

Rat Leydig cells were prepared from adult male Sprague-Dawley rats and purified and cultured for $20 \mathrm{~h}$ as described by Sharpe \& Cooper (1984) and Risbridger et al. (1986). Briefly, purified Leydig cells were obtained using discontinuous gradients of Percoll (Pharmacia Ltd, Uppsala, Sweden). Test substances were incubated with $5 \times 10^{4}$ purified Leydig cells for $16-20 \mathrm{~h}$ in plastic multiwell dishes (Costar, Cambridge, MA, U.S.A.) in a final volume of $250 \mu$ l. The cultures were stored at $-20^{\circ} \mathrm{C}$ until assayed for testosterone. Testicular interstitial fluid was collected from adult male 
rats made bilaterally cryptorchid for 15 months, and charcoal treated as described by Sharpe \& Cooper (1984). The pool of interstitial fluid thus obtained stimulated Leydig cell testosterone production in a dose-dependent manner similar to that from normal adult rats, although it was more potent (Fig. 1).

Medium M199E was obtained from Flow Laboratories (McLean, VA, U.S.A.) and hCG (Pregnyl) was obtained from Organon (Cambridge, U.K.). (25R)-25-Hydroxycholesterol and (22R)-22-hydroxycholesterol (Sigma, St Louis, MO, U.S.A.) were dissolved in ethanol and diluted in medium to the appropriate concentration. Cycloheximide (Sigma) was dissolved in ethanol and added at a final concentration of $10 \mu \mathrm{l} / \mathrm{ml} \mathrm{M} 199 \mathrm{E}$ or $0.1 \mathrm{mM}$. Aminoglutethimide was obtained from Ciba Geigy, Sussex, U.K. Cyanoketone and SU 10603, compounds which inhibit $3 \beta$ hydroxysteroid dehydrogenase and $17 \alpha$-hydroxylase respectively, were obtained from $\mathrm{Dr} F$. Rommerts, Erasmus University, Rotterdam, The Netherlands.

Testosterone concentrations in the incubation media were measured directly using a $\left[{ }^{3} \mathrm{H}\right]$ testosterone RIA as described by Risbridger, Kerr \& de Kretser (1981) and an antiserum raised in sheep against testosterone 3-carboxymethyloxime coupled to bovine serum albumin. The antiserum showed a $13.6 \%$ cross-reactivity with $5 \alpha$-dihydrotestosterone. The sensitivity of the assay was $<0.079 \mathrm{ng} / \mathrm{ml}(n=7)$, the interassay coefficient of variation was $7.9 \%(n=7)$ and the intra-assay coefficient of variation was $5.7 \%(n=7)$. Pregnenolone concentrations were determined in medium by a direct radioimmunoassay using an antiserum raised against pregnenolone-3-monohemisuccinate-HSA (Radioassay Systems Laboratories, Carson, California, U.S.A., 1:2000 initial dilution), $\left[7-{ }^{3} \mathrm{H}(\mathrm{n})\right]$ pregnenolone (sp. act. $19 \cdot 3 \mathrm{Ci} / \mathrm{mmol}, 10000$ c.p.m., NEN, Boston, MA, U.S.A.) and pregnenolone standard (Sigma). The antiserum cross-reacted $100 \%$ with pregnenolone, $50 \%$ with pregnenolone sulphate, $2 \%$ with 17 -hydroxyprogesterone and $1.5 \%$ with progesterone. Pregnenolone was undetectable in charcoal-treated interstitial fluid, and standard curves prepared using $100 \mu \mathrm{l}$ interstitial fluid instead of buffer diluted in parallel with the buffer standard curve. The sensitivity of the assay was $12.4 \pm 1 \cdot 7$ (s.e.m.) pg/tube $(n=10)$, the interassay coefficient of variation was $13.5 \%(n=21)$ and the intra-assay coefficient of variation was $11.2 \%$ $(n=13)$.

Results were analysed for statistical difference using Student's $t$ test, or one way analysis of variance and Duncan's Multiple Range Test.

\section{Results}

Maximum stimulation of testosterone production in vitro by hCG was achieved with at least $0.25 \mathrm{mi}$.u. hCG per incubation well (Fig. 2). In the presence of $2.5 \mathrm{i} . \mathrm{u}$. hCG, the addition of 3-50 $\mu \mathrm{l}$ rat interstitial fluid caused a further and significant $(P<0.01)$ stimulation of testosterone production (Fig. 1).

The addition of 25- and 22-hydroxycholesterol enhanced basal and hCG-stimulated steroidogenesis by Leydig cells in vitro although more testosterone was formed from 22-hydroxycholesterol (Fig. 3). With the addition of the higher concentrations of hydroxycholesterol, testosterone production reached the same level irrespective of whether or not hCG was present. Interstitial fluid alone stimulated testosterone output, although the dose-response line was not parallel $(P>0.01)$ to that observed with 22-hydroxycholesterol (Fig. 4), indicating that the action of these two substances is dissimilar. This was confirmed by the addition of cycloheximide which did not affect the metabolism of hydroxycholesterol, but blocked the stimulatory action of interstitial fluid (Fig. 4). Increasing volumes of interstitial fluid did not stimulate testosterone production in the presence of $20 \mu \mathrm{M}$-22-hydroxycholesterol and hCG (Fig. 5). However, pregnenolone production was increased in a dose-dependent manner, indicating that, with these high levels of 22 -hydroxycholesterol and hCG, the enzymes required to metabolize pregnenolone to testosterone had become limiting. These results suggested that interstitial fluid acted at a step before the conversion of pregnenolone. 

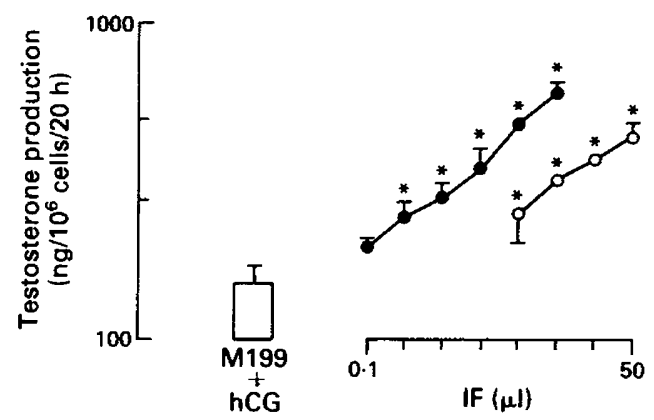

Fig. 1. The effect of testicular interstitial fluid (IF) obtained from intact $(O)$ or long-term cryptorchid (O) rats on hCG-stimulated testosterone production by Leydig cells in vitro. Each point represents the mean \pm s.d. $(n=3) .{ }^{*} P<0.05$ compared to testosterone production in Medium M199 + hCG.

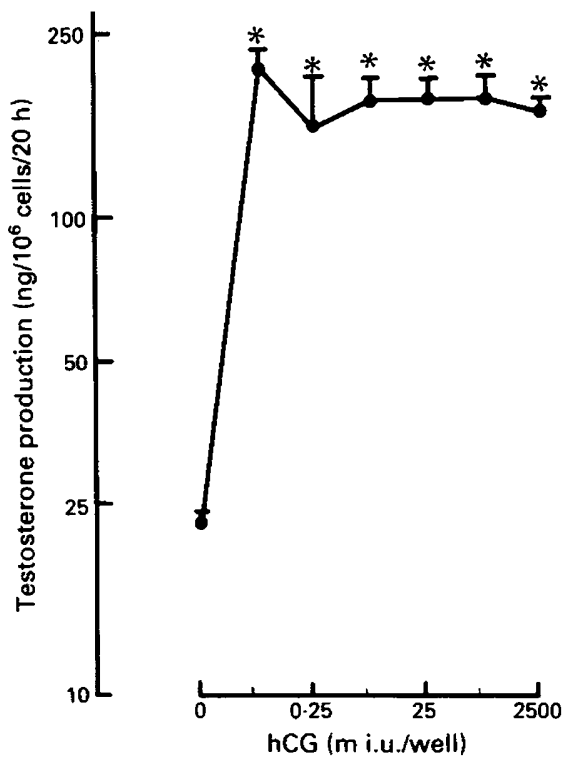

Fig. 2. The effect of increasing doses of hCG on testosterone production in vitro by Leydig cells. Values are mean \pm s.d. $(n=3)$. ${ }^{*} P<0.01$ compared to unstimulated testosterone production.

The site of action of rat interstitial fluid on testicular steroidogenesis was then investigated by using inhibitors of 3 $\beta$-hydroxysteroid dehydrogenase (SU 10603) and 17 $\alpha$-hydroxylase (cyanoketone). The stimulation of testosterone production in the presence of hCG, interstitial fluid or hCG + interstitial fluid was blocked by the addition of these inhibitors, but the concentrations of pregnenolone were significantly increased (Fig. 6). The addition of aminoglutethimide which inhibits an enzyme step before the conversion of pregnenolone also inhibited the stimulatory effects of hCG, interstitial fluid or hCG + interstitial fluid (Fig. 7). 
Fig. 3

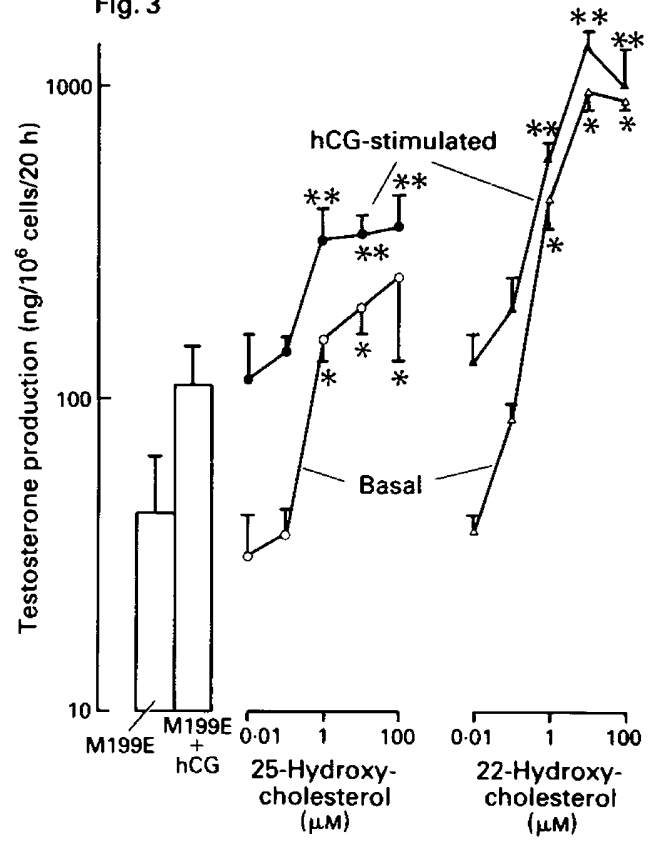

Fig. 4

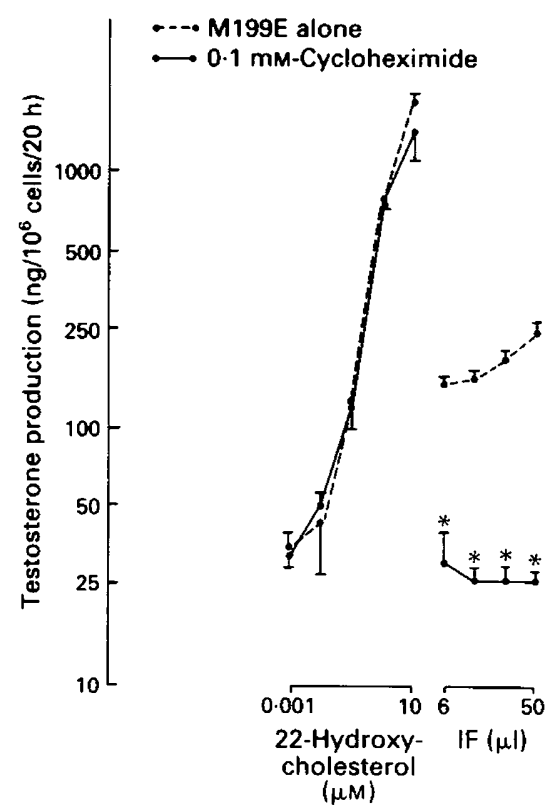

Fig. 3. The effect of 25- and 22-hydroxycholesterol on basal and hCG-stimulated testosterone production in vitro by isolated Leydig cells. Values are mean \pm s.d. $(n=3)$. ${ }^{*} P<0.05$ compared to testosterone produced in Medium M199E; $* * P<0.05$ compared to testosterone produced in Medium M199E + hCG.

Fig. 4. The effect of 22-hydroxycholesterol or interstitial fluid (IF) on testosterone production in vitro by Leydig cells in the absence or presence of $0.1 \mathrm{mM}$-cycloheximide. Values are mean \pm s.d. $(n=3) .{ }^{*} P<0.05$ compared to testosterone produced in Medium M199E alone.

\section{Discussion}

These results demonstrate that testicular interstitial fluid contains a factor that stimulates testosterone production before the formation of pregnenolone. The mechanism by which testosterone production is stimulated is dissimilar to that obtained by the addition of hydroxycholesterol and indicates that interstitial fluid does not simply act by providing substrate for metabolism to testosterone.

It has been reported that steroid production by the ovary and testis is enhanced in the presence of hydroxy steroids (Bakker, van der Plank/van Winsen \& van der Molen, 1979; Taoff, Schleyer \& Strauss, 1982). Our results confirm these findings and agree with those of Sullivan \& Cooke (1983) which show that more testosterone was formed from the metabolism of 22-hydroxycholesterol than from 25-hydroxycholesterol. The suggestion by Taoff et al. (1982) that not enough endogenous substrate is available or can be mobilized to achieve the highest rates of steroidogenesis in vitro is supported by the fact that testosterone production was increased by the addition of hydroxycholesterol in the presence of a maximally stimulating dose of hCG. Furthermore, in the absence of hCG, the same levels of testosterone were achieved by the addition of hydroxycholesterol alone.

The addition of interstitial fluid also enhanced Leydig cell steroidogenesis in vitro, but this stimulatory action was inhibited in the presence of cycloheximide, an inhibitor of protein synthesis. 


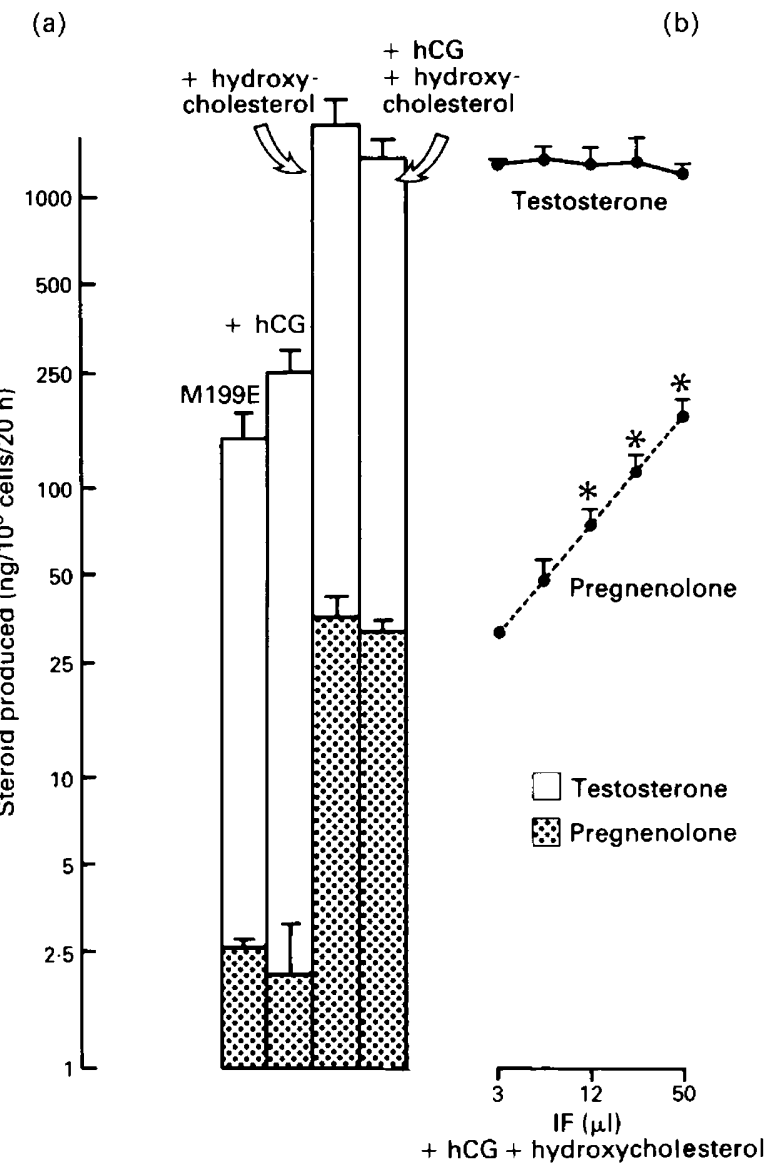

Fig. 5. The effect of hCG, 22-hydroxycholesterol and interstitial fluid (IF) on Leydig cell steroidogenesis in vitro. (a) Testosterone and pregnenolone production in the presence of Medium M199E \pm hCG (2.5 i.u.) \pm hydroxycholesterol $(20 \mu \mathrm{M})$. (b) Testosterone and pregnenolone production in the presence of Medium M199E + hCG + hydroxycholesterol and increasing volumes of interstitial fluid (IF). Values are mean \pm s.d. $(n=3)$. ${ }^{*} P<0.05$ compared to pregnenolone produced in the presence of $\mathrm{hCG}+$ hydroxycholesterol alone.

In contrast the metabolism of 22-hydroxycholesterol to testosterone by Leydig cells was not affected by cycloheximide. Interstitial fluid therefore does not provide the cells with cholesterol for metabolism to testosterone. The addition of interstitial fluid to Leydig cells incubated in the presence of hydroxycholesterol did not cause a dose-dependent increase in testosterone production. This result could have been due to a limitation of one of the enzymes involved in the steroidogenic pathway, but measurement of pregnenolone values in the same samples revealed a dose-dependent increase in the concentrations of this steroid with increasing volumes of interstitial fluid. It is therefore concluded that, under these conditions, the enzymes responsible for the metabolism of pregnenolone to testosterone had become rate-limiting. These results demonstrate that interstitial fluid stimulates steroidogenesis, before the conversion of pregnenolone. This was confirmed by the ability of the enzyme inhibitors SU 10603 and cyanoketone (which inhibit the steroidogenic enzymes $3 \beta$-hydroxysteroid dehydrogenase and $17 \alpha$-hydroxylase respectively) to block interstitial fluid stimulation of testosterone production in the presence of hCG. The resultant accumulation of pregnenolone is significantly greater in the presence of interstitial fluid and hCG compared to hCG 


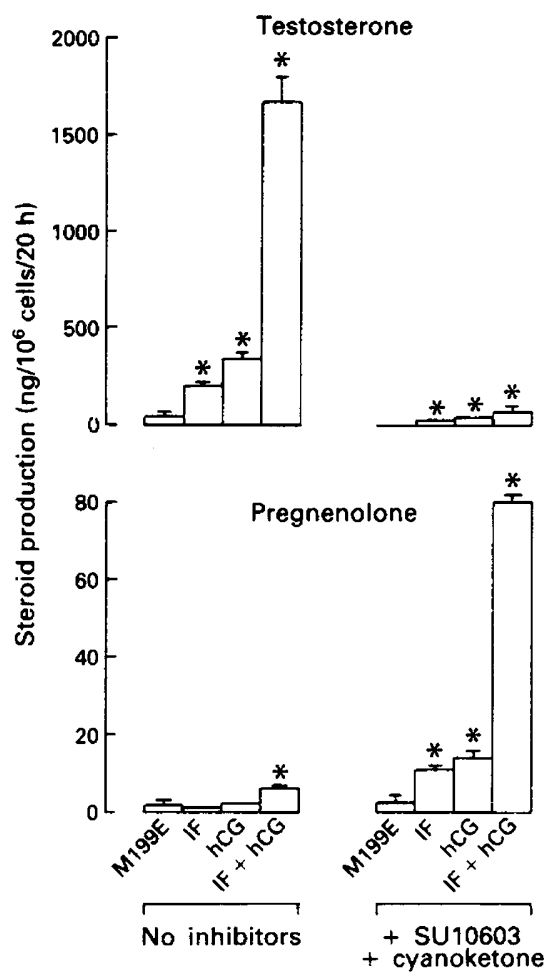

Fig. 6. The effect of SU 10603 and cyanoketone on Leydig cell steroidogenesis stimulated by hCG (2.5 i.u.), interstitial fluid $(25 \mu \mathrm{l})$ or hCG + interstitial fluid. Values are mean \pm s.d. $(n=3){ }^{*} P<0.01$ compared to steroid production in Median M199E. Note that the vertical axis is an arithmetic scale.

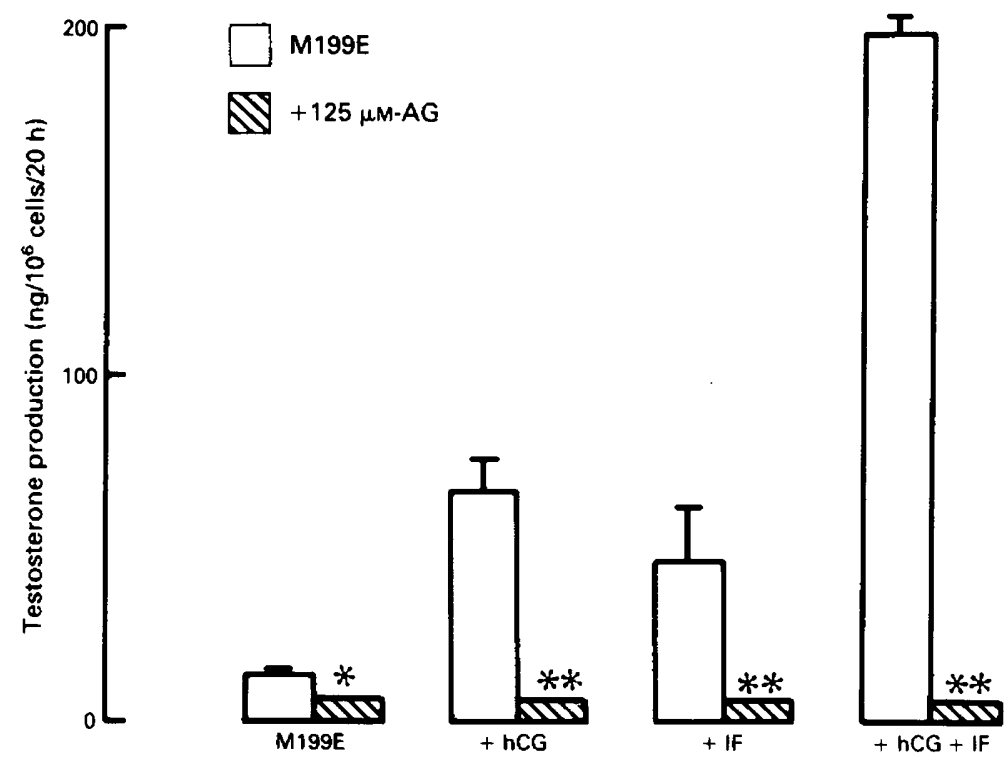

Fig. 7. The effect of aminoglutethimide on testosterone production by isolated Leydig cells stimulated with hCG $(2.5$ i.u.), interstitial fluid (IF, $25 \mu \mathrm{l})$ or hCG + IF. Values are mean \pm s.d. $(n=3){ }^{*} P<0.05$ compared to steroid production in Medium M199E. Note that the vertical axis is an arithmetic scale. 
alone, demonstrating again that interstitial fluid stimulates steroidogenesis before the formation of pregnenolone. In confirmation of these data, aminoglutethimide, which inhibits steroidogenesis before pregnenolone production (Dexter, Fishman, Ney \& Liddle, 1967) abolishes the stimulatory action of interstitial fluid.

The rate-limiting step in the synthesis of testosterone by Leydig cells is the cleavage of cholesterol to pregnenolone by the cholesterol side-chain cleavage enzyme (Hall, 1970). The rate of cholesterol sidechain cleavage (as well as the transport of cholesterol to the mitochondria) is also increased by the trophic stimulus of $\mathrm{LH}$ (or hCG) which is the principal hormone regulating Leydig cell testosterone synthesis. However, the stimulatory activity in testicular interstitial fluid is not due to the presence of pituitary LH (Sharpe \& Cooper, 1984). Nevertheless, it is reasonable to propose that, if the factor in testicular interstitial fluid has a physiological role to play in the local regulation of Leydig cell steroidogenesis, it would act to stimulate this rate-limiting step of steroidogenesis. The results presented in this paper are entirely consistent with the hypothesis that interstitial fluid acts to stimulate the conversion of cholesterol to pregnenolone.

In addition to those demonstrating that the activity of the interstitial fluid factor(s) increases when intratesticular concentrations of testosterone are reduced or vice versa (Sharpe \& Cooper, 1984; Sharpe \& Bartlett, 1985), these results indicate a fundamental role of the factor in the paracrine regulation of testicular function.

This study was supported by NH and MRC, Australia. We thank J. A. Muir and M. Lawford for assistance.

\section{References}

Bakker, C.P., van der Plank/van Winsen, M.P.I. \& van der Molen, H.J. (1979) Effect of cholesterol, hydroxycholesterols and calcium on pregnenolone production rates in mitochondrial fractions from rat testis. Biochem. Biophys. Acta 584, 94-103.

de Kretser, D.M. (1982) Intratesticular regulatory mechanisms. Int. J. Androl., Suppl. 5, 11-17.

Dexter, R.N., Fishman, L.M., Ney, R.L. \& Liddle, G.W. (1967) Inhibition of adrenal corticosteroid synthesis by aminoglutethimide: studies of the mechanism of action. J. clin. Endocr. Metab. 27, 473-480.

Hall, P.F. (1970) Endocrinology of the testis. In The Testis, vol. 2, pp. 1-71. Eds A. D. Johnson, W. R. Gomes \& N. L. VanDemark. Academic Press, New York.

Parvinen, M. (1982) Regulation of the seminiferous epithelium. Endocr. Rev. 3, 404-417.

Risbridger, G.P., Kerr, J.B. \& de Kretser, D.M. (198I) Evaluation of Leydig cell function and gonadotrophin binding in unilateral and bilateral cryptorchidism: evidence of local control of Leydig cell function by the seminiferous tubule. Biol. Reprod. 24, 534-540.
Risbridger, G.P., Muir, J.A., Robertson, D.M. \& de Kretser, D.M. (1986) Evidence for a novel factor in testicular interstitial fluid capable of stimulating testosterone production by rat Leydig cells. Molec. cell. Endocrinol. (in press).

Sharpe, R.M. (1983) Local control of testicular function. Q. Jl exp. Physiol. 68, 265-287.

Sharpe, R.M. \& Bartlett, J.M.S. (1985) Intratesticular distribution of testosterone in rats and the relationship to the concentrations of a peptide that stimulates testosterone secretion. J. Reprod. Fert. 73, 223-236.

Sharpe, R.M. \& Cooper, I. (1984) Intratesticular secretion of a factor(s) with major stimulatory effects on Leydig cell testosterone secretion in vitro. Molec. cell. Endocrinol. 37, 159-168.

Sullivan, M.J.F. \& Cooke, B.A. (1983) Stimulation of cholesterol side-chain cleavage by a luteinizinghormone-releasing hormone (luliberin) agonist (ICI 118630 ) in rat Leydig cells. Biochem. J. 216, 747-752.

Taoff, M.E., Schleyer, H. \& Strauss, J.F. (1982) Metabolism of 25-hydroxycholesterol by rat luteal mitochondria and dispersed cells. Endocrinology 111 , 1785-1790. 